\title{
Modular discretization of the $\mathrm{AdS}_{2} / \mathrm{CFT}_{1}$ holography
}

\author{
Minos Axenides, ${ }^{a}$ Emmanuel Floratos ${ }^{a, b, c}$ and Stam Nicolis ${ }^{d}$ \\ ${ }^{a}$ Institute of Nuclear and Particle Physics, N.C.S.R. Demokritos, \\ GR-15310, Athens, Greece \\ ${ }^{b}$ Department of Physics, University of Athens, \\ GR-157r1 Athens, Greece \\ ${ }^{c}$ Theory Division, Department of Physics, CERN, \\ CH-1211 Geneva 23, Switzerland \\ ${ }^{d}$ CNRS-Laboratoire de Mathématiques et Physique Théorique (UMR 7350), \\ Fédération 'Denis Poisson' (FR2964), Université de Tours 'François Rabelais', \\ Parc Grandmont, 37200 Tours, France \\ E-mail: axenides@inp.demokritos.gr, mflorato@phys.uoa.gr, \\ Stam.Nicolis@lmpt.univ-tours.fr
}

ABSTRACT: We propose a finite discretization for the black hole, near horizon, geometry and dynamics. We realize our proposal, in the case of extremal black holes, for which the radial and temporal near horizon geometry is known to be $\operatorname{AdS}_{2}=\operatorname{SL}(2, \mathbb{R}) / \mathrm{SO}(1,1, \mathbb{R})$. We implement its discretization by replacing the set of real numbers $\mathbb{R}$ with the set of integers modulo $N$ with $\mathrm{AdS}_{2}$ going over to the finite geometry $\operatorname{AdS}_{2}[N]=\operatorname{SL}\left(2, \mathbb{Z}_{N}\right) / \operatorname{SO}\left(1,1, \mathbb{Z}_{N}\right)$. We model the dynamics of the microscopic degrees of freedom by generalized Arnol'd cat maps, $\mathrm{A} \in \mathrm{SL}\left(2, \mathbb{Z}_{N}\right)$ which are isometries of the geometry, at both the classical and quantum levels. These are well known to exhibit fast quantum information processing through the well studied properties of strong arithmetic chaos, dynamical entropy, nonlocality and factorization in the cutoff discretization $N$. We construct, finally, a new kind of unitary and holographic correspondence, for $\operatorname{AdS}_{2}[N] / \mathrm{CFT}_{1}[N]$, via coherent states of the bulk and boundary geometries.

KEYwords: Black Holes in String Theory, AdS-CFT Correspondence

ARXIV EPRINT: 1306.5670 


\section{Contents}

1 Introduction 1

2 Observers, geometry of cosets and Weyl dynamics on $\mathrm{AdS}_{2} \quad 4$

3 Modular discretization and quantum dynamics on $\mathrm{AdS}_{2}[N] \quad 12$

4 The coherent states of bulk and boundary 16

$5 \operatorname{AdS}_{2}[N] / \mathrm{CFT}_{1}[N]$ coherent state holography 21

$\begin{array}{llr}6 & \text { Summary and conclusions } & 24\end{array}$

\section{Introduction}

From the early days of the black hole information paradox it has been suggested that, in order to bring together quantum mechanics and the equivalence principle consistently, we have to give up locality and the semiclassical description in the near horizon region. Key ingredients in the framework, that is likely to replace them, include the principle of holography [1, 2], complementarity [3] and strongly chaotic dynamics [4]. Recent discussions on the nature and the dynamics of microscopic degrees of freedom in the near horizon region of black holes have, also, strengthened the conviction that semiclassical physics is inadequate to guarantee the compatibility of the equivalence principle and quantum mechanics. Therefore it seems imperative that a drastic departure from conventional, semiclassical, physics is needed [5].

We should stress here that recent developments in string theory [6-10], which take into account the correct definition of the black hole entropy at the quantum level, have improved considerably our understanding of the black hole microscopic degrees of freedom. We understand now some important quantum statistical properties, in particular, the exact quantum entropy, for a certain class of extremal black holes.

Important issues have, yet, remained open, which dominate the recent literature. They pertain to the description of the nature and the dynamics of the near horizon microstates.

In what follows we consider the simplest dynamical context for the discussion of the black hole information paradox [11]. Consider two observers, one at infinity, $O_{a}$ and the other in free fall, $O_{b}$ into the black hole. The question is, if there exists a unitary transformation, connecting the description of a freely falling particle near the horizon by the two observers.

In the seminal paper [12] the corresponding quantum mechanical evolution operators have been constructed, by two, different, conformal invariant, Hamiltonians. The "first" 
Hamiltonian, which, in our case, describes the time evolution for observer $O_{a}$, has continuous spectrum, while the "second" Hamiltonian, which describes the time evolution for observer $O_{b}$, in our case, has discrete spectrum.

There has, also, been extensive discussion in the literature, how these two descriptions can be related to the paradox between the infinite number of states near the horizon thus described and the finite entropy of the black hole, which is proportional to its area [13-17]. Though developments in string theory (reviewed in $[6,7]$ ), around the same time, succeeded in resolving the problem, of counting these states and of reproducing the thermodynamical result for the black hole entropy, how to extend this calculation for the case of a "small" number of microstates, i.e. for "small" black holes, remains open $[6,7]$.

The information paradox, in the case of extremal black holes, which have an $\mathrm{AdS}_{2}$ radial and temporal, near horizon, geometry, is expressed by the putative mismatch between the description accessible to observer, $O_{a}$, on the boundary, using a $\mathrm{CFT}_{1}$ dynamics and that of the, free-falling, observer, $O_{b}$, who is using the bulk, $\mathrm{AdS}_{2}$, dynamics. The resolution of this paradox is currently the subject of intense research activity [18-23].

In the present and subsequent works we study the above issues using a space-time discretization of the near horizon geometry, $\operatorname{AdS}_{2}=\mathrm{SL}(2, \mathbb{R}) / \mathrm{SO}(1,1, \mathbb{R})$, of extremal black holes. We assume it to be discrete and finite as a consequence of the finite dimension of the Hilbert space of black hole microstates. Indeed, the existence of a finite number, of linearly independent wavefunctions, as probes, implies a finite resolution of its spacetime geometry. This is achieved, simply, by replacing the set of real numbers $\mathbb{R}$ by the set of integers modulo $N, \mathbb{Z}_{N}$, for any positive integer $N$. The discretization thus replaces the continuous spacetime $\mathrm{AdS}_{2}$ by the finite arithmetic geometry, $\operatorname{AdS}_{2}[N]=\mathrm{SL}\left(2, \mathbb{Z}_{N}\right) / \mathrm{SO}\left(1,1, \mathbb{Z}_{N}\right)[24,25]$.

This discretization, which we call "modular discretization", has the merit of preserving corresponding symmetry properties of the continuous, classical, space-time geometry and provides a means of defining a consistent quantum geometry, through holography. In this discretized setting we will construct the corresponding unitary evolution operators for the two observers.

The discretization defines an infrared cutoff $L$ as well as a UV one $\frac{L}{N}$. By considering the $N^{2}$ points of an $L \times L$ square lattice and lifting to the $\mathrm{AdS}_{2}$ by stereographic projection, we obtain a spacetime discretization. It is obvious that the continuum limit can be recovered by taking first the $N \rightarrow \infty$ limit at fixed $L$ and, afterwards, the limit $L \rightarrow \infty$. It is important to stress at this point the independence of the cutoff, $L$ from the $\mathrm{AdS}_{2}$ radius $R_{\mathrm{AdS}_{2}}$.

In order to describe the dynamics of probes, at both the classical and quantum level, we use the Arnol'd cat maps, A, elements of $\mathrm{SL}\left(2, \mathbb{Z}_{N}\right)$. They are known to possess properties of strong arithmetic chaos, ergodic mixing and locality [26-28]. These maps also satisfy factorization properties in the discretization cutoff $N$, which induce fast quantum information processing between the probe and the near horizon geometry [29, 30].

Our present work builds on our earlier work on Finite Quantum Mechanics (FQM) where we introduced the discretized toroidal membrane, $\mathbb{Z}_{N} \times \mathbb{Z}_{N}$ [31] which elucidates the matrix model truncation of the membrane dynamics [32, 33] rendering the discrete membrane as a quantum phase space of finite quantum mechanics, with canonical transformation group $\mathrm{SL}\left(2, \mathbb{Z}_{N}\right)[34-38]$. 
Interestingly enough, the discretized membrane, in the black hole setting, describes the geometry of the stretched horizon [11] and the matrix model describes the M-theoretic dynamics of its microscopic degrees of freedom [4].

We extend these results to the case of the $\operatorname{AdS}_{2}[N]$, discrete, near horizon, bulk geometry, and the dynamics of the infalling observer, $O_{b}$, along with its associated boundary $\mathrm{CFT}_{1}[N]$ and the observer $O_{a}$ in order to obtain a holographic correspondence. In the discrete case the boundary is constructed as a coset space $\operatorname{SL}\left(2, \mathbb{Z}_{N}\right) / \mathfrak{B}_{N}$, which is identified with the discrete projective line, $\mathbb{R P}_{N}^{1}$. Here $\mathfrak{B}_{N}$ is the Borel subgroup of $\operatorname{SL}\left(2, \mathbb{Z}_{N}\right)$, which fixes the point at infinity.

The group $\operatorname{SL}\left(2, \mathbb{Z}_{N}\right)$ plays three different roles in the present context: a)as the isometry group of $\mathrm{AdS}_{2}[N]$, b) as the symplectic group of $\mathrm{AdS}_{2}[N]$ which is considered to be a (stringy) phase space and c) as the conformal group of the boundary. (a) and (c) are the basic reasons for the existence of the $\operatorname{AdS}_{2}[N] / \mathrm{CFT}_{1}[N]$ correspondence and (b) is going to be used for the quantization of the geometry and the dynamics.

We construct the discrete time evolution, quantum unitary maps explicitly and discuss their action on the common $N$-dimensional Hilbert space of both the bulk and the boundary. The natural action of these quantum maps is realized on the set of coherent states, appropriate for the bulk and boundary coset geometries. These states inherit classical chaotic dynamics, define isometric invariant (bulk-bulk,bulk-boundary and boundaryboundary) propagators and are convenient for the discussion of localization problems in the AdS/CFT correspondence, since they saturate the uncertainty relation of the UV/IR connection [39-44].

The plan of the present work is as follows:

In section 2 we review the construction of the smooth $\mathrm{AdS}_{2}$ geometry and its boundary, as a doubly ruled surface by rotating the light cone lines around the time-like circle. We establish various global coordinate systems, through appropriate coset parametrizations. More specifically we show that the light cone coordinates, on the stereographic projection plane, parametrize holographically both the bulk and its boundary.

In order to describe the high energy dynamics for the the radial motion of probes, we employ linear isometry maps, $A \in \mathrm{SL}(2, \mathbb{R})$, which are appropriate for the description of the infalling (bulk) and static (boundary) observers.

In section 3 we motivate the introduction of the arithmetic discretization $\bmod N$. We define the Finite Quantum Mechanics for both the bulk and the boundary on the same Hilbert space. We shall work in the Hilbert space of the metaplectic representation of $\operatorname{SL}\left(2, \mathbb{Z}_{N}\right)$ of dimension $N$ for the simplest case $N=p$ of an odd prime. In this case $Z_{N}=\mathbb{F}_{p}$ is the simplest Galois field.

The methods to be presented apply also for all other irreps of this group. In the case $N=p$, an odd prime, the number of irreps is $p+4$. They have been worked out in detail, using the induced representations, and the corresponding multiplicative characters of $\mathbb{F}_{p}$, or $\mathbb{F}_{p^{2}}[45]$.

The boundary is also constructed as a coset space $\operatorname{SL}\left(2, \mathbb{F}_{p}\right) / \mathfrak{B}_{p}$, which is identified with the discrete projective line, $\mathbb{R P}_{p}^{1}$. Here $\mathfrak{B}_{p}$ is the Borel subgroup of $\operatorname{SL}\left(2, \mathbb{F}_{p}\right)$, which fixes the point at infinity. 
In section 4 we explicitly construct the bulk and the boundary overcomplete set of discrete coherent states. We discuss their basic properties as they are appropriate for the corresponding coset geometries.

The states and the observables are also expanded on the coherent states and their time evolution is defined through the quantum cat maps. The correlation functions of various observables are defined, as well as the method of their evaluation.

Finally, in section 5, we exhibit the reconstruction (holography) of the bulk coherent states from those of the boundary, via the bulk-boundary, bulk-bulk, boundary-boundary propagators and the consequent reconstruction of the scalar bulk observables from the boundary ones. The correlation functions of scalar observables in the bulk and the boundary are connected through this holography and can be explicitly calculated.

In the last section 6 we summarize our results and their role in the context of the problem of black hole information processing. We also comment on future work on the complete description of finite conformal quantum mechanics on the boundary as well as on how the scrambling time bound might be saturated [4].

\section{Observers, geometry of cosets and Weyl dynamics on $\mathrm{AdS}_{2}$}

Consider the dynamics of freely falling bodies, in the near horizon region of spherically symmetric $4 \mathrm{~d}$ extremal black holes . The geometry is known to be of the form $\mathrm{AdS}_{2} \times S^{2}$, where the $\mathrm{AdS}_{2}=\mathrm{SL}(2, \mathbb{R}) / \mathrm{SO}(1,1, \mathbb{R})$, factor describes the geometry of the radial and time coordinates and $S^{2}$ is the horizon surface. We will compare the description of high energy radial dynamics as seen by (radial) observers(static or freely falling), for which the transverse and longitutinal motion is decoupled.

To each of these observers corresponds a global space-time coordinate system and in the following we shall exhibit some of them using group theory.

The $\mathrm{AdS}_{2}$ spacetime, is a one-sheeted hyperboloid defined through its global embedding in Minkowski spacetime with one space - and two time-like dimensions by the equation $[46,47]$.

$$
x_{0}^{2}+x_{1}^{2}-x_{2}^{2}=1
$$

The boundaries of $\mathrm{AdS}_{2}$ consist of two time-like disconnected circles, where $\mathrm{AdS}_{2}$ approaches, asymptotically, the light cone of $\mathscr{M}^{1,2}$,

$$
x_{0}^{2}+x_{1}^{2}-x_{2}^{2}=0
$$

$\mathrm{AdS}_{2}$ is, at the same time, the homogeneous space, $\mathrm{SO}(1,2) / \mathrm{SO}(1,1)$. This case, is special, in that $\mathrm{SO}(1,2)$ has a double cover, $\mathrm{SL}(2, \mathbb{R})$, so we have $\mathrm{AdS}_{2}=\mathrm{SL}(2, \mathbb{R}) / \mathrm{SO}(1,1)$.

In order to establish our notation and conventions, we proceed with the Weyl construction of the double covering group, $\operatorname{SL}(2, \mathbb{R})$.

To every point, $x_{\mu} \in \mathrm{AdS}_{2}, \mu=0,1,2$, we assign the traceless, real, $2 \times 2$ matrix

$$
\mathrm{M}(x) \equiv\left(\begin{array}{cc}
x_{0} & x_{1}+x_{2} \\
x_{1}-x_{2} & -x_{0}
\end{array}\right)
$$

Its determinant is, $\operatorname{det} \mathrm{M}(x)=-x_{0}^{2}-x_{1}^{2}+x_{2}^{2}=-1$. 
The action of any $\mathrm{A} \in \mathrm{SL}(2, \mathbb{R})$ on $\mathrm{AdS}_{2}$ is defined through the non-linear mapping

$$
\mathrm{M}\left(x^{\prime}\right)=\mathrm{AM}(x) \mathrm{A}^{-1}
$$

This induces an $\mathrm{SO}(1,2)$ transformation on $\left(x_{\mu}\right)_{\mu=0,1,2}$,

$$
x^{\prime} \equiv \mathrm{L}(\mathrm{A}) x
$$

Choosing, as the origin of coordinates, the base point $\boldsymbol{p} \equiv(1,0,0)$, its stability group $\mathrm{SO}(1,1)$ is the group of Lorentz transformations in the $x_{0}=0$ plane of $\mathscr{M}^{1,2}$ or equivalently, the "scaling" subgroup, D, of $\mathrm{SL}(2, \mathbb{R})$

$$
\mathrm{D} \ni \mathbf{S}(\lambda) \equiv\left(\begin{array}{cc}
\lambda & 0 \\
0 & \lambda^{-1}
\end{array}\right)
$$

for $\lambda \in \mathbb{R}^{*}$.

For this choice of the stability point, we define the coset, $h_{\mathrm{A}}$, by decomposing $\mathrm{A}$ as

$$
\mathrm{A}=h_{\mathrm{A}} \mathrm{S}\left(\lambda_{\mathrm{A}}\right)
$$

Thus, we associate uniquely to every point $x \in \mathrm{AdS}_{2}$ the corresponding coset representative $h_{\mathrm{A}}(x)$.

We introduce now, the global coordinate system, defined by the straight lines that generate $\mathrm{AdS}_{2}$ and for which it can be checked easily that they form its complete set of light cones.

Consider the two lines, $\boldsymbol{l}_{ \pm}(\boldsymbol{p})$, passing through the point $\boldsymbol{p} \in \mathscr{M}^{1,2}$, orthogonal to the $x_{0}$ axis and at angles $\pm \pi / 4$ to the $x_{1}=0$ plane. They are defined by the intersection of $\mathrm{AdS}_{2}$ and the plane $x_{0}=1 \mathrm{cf}$. figure 1 .

The coordinates of any point, $\boldsymbol{q}_{+} \in \boldsymbol{l}_{+}(\boldsymbol{p}), \boldsymbol{q}_{-} \in \boldsymbol{l}_{-}(\boldsymbol{p})$ are given as, $\left(1, \mu_{ \pm}, \pm \mu_{ \pm}\right)$, $\mu_{ \pm} \in \mathbb{R}$ correspondingly.

Rotating these lines, around the $x_{0}, x_{1}$ time circle, by appropriate angles $\phi_{ \pm} \in[0,2 \pi)$, we can parametrize any point by their inersection, with coordinates

$$
\begin{aligned}
& x_{0}=\cos \phi_{ \pm}-\mu_{ \pm} \sin \phi_{ \pm} \\
& x_{1}=\sin \phi_{ \pm}+\mu_{ \pm} \cos \phi_{ \pm} \\
& x_{2}= \pm \mu_{ \pm}
\end{aligned}
$$

The corresponding pair of crossing lines, $\boldsymbol{l}_{ \pm}(\boldsymbol{x})$, define the local light cone.

Another form of the previous equation is:

$$
e^{\mathrm{i} \phi_{ \pm}}=\frac{x_{0} \pm \mathrm{i} x_{1}}{1 \pm x_{2}} \quad \mu_{ \pm}= \pm x_{2}
$$

The corresponding coset parametrization (group coset motion which brings the origin to the point $x$ ) is:

$$
h\left(\mu_{ \pm}, \phi_{ \pm}\right)=\mathrm{R}\left(\phi_{ \pm}\right) \mathrm{T}_{ \pm}\left(\mu_{ \pm}\right)
$$




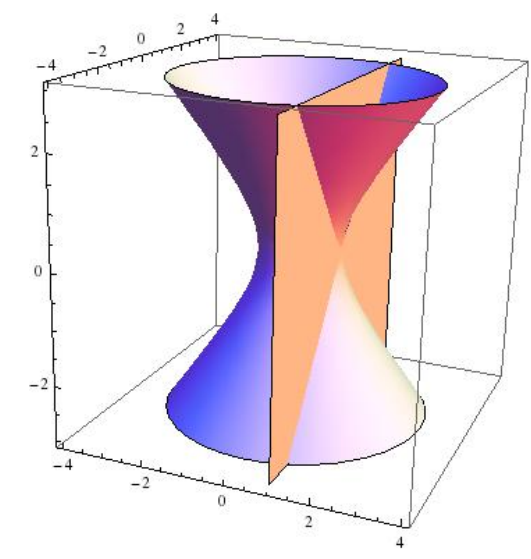

Figure 1. The light cone of $\mathrm{AdS}_{2}$ at $\boldsymbol{p}=(1,0,0)$.

where

$$
\mathrm{R}(\phi)=\left(\begin{array}{cc}
\cos \phi / 2 & -\sin \phi / 2 \\
\sin \phi / 2 & \cos \phi / 2
\end{array}\right)
$$

and

$$
\mathrm{T}_{+}(\mu)=\left[\mathrm{T}_{-}(-\mu)\right]^{\mathrm{T}}=\left(\begin{array}{cc}
1 & -\mu \\
0 & 1
\end{array}\right)
$$

It is easy to see also, that $\mathrm{T}_{ \pm}\left(\mu_{ \pm}\right)$, acting on the base point $X(\boldsymbol{p})$, generate the light cone $l_{ \pm}(\boldsymbol{p})$, so we identify these one parameter subgroups with the light cones at $p$. At this point we should like to pause and discuss the physical interpretation of the rotation (2.11) and translation (2.12) groups.

Consider two observers, in the $\mathrm{AdS}_{2}$ background, one at infinity, $O_{a}$ and the other in free fall, $O_{b}$. Their corresponding classical description for a freely falling particle near the horizon, is given by the finite group of translations, with time parameter $\mu$ for $O_{a}-$ and rotations, with time parameter $\phi$ for $O_{b}$. In the seminal paper [12] the corresponding quantum mechanical evolution operators have been constructed in order to describe models for confinement and asymptotic freedom, by two, different, conformal invariant, Hamiltonians. The "first" Hamiltonian, which, in our case, describes the time evolution for observer $O_{a}$, has continuous spectrum, while the "second" Hamiltonian, which describes the time evolution for observer $O_{b}$, in our case, has discrete spectrum.

There has been extensive discussion in the literature how these two descriptions can be related to the paradox between the infinite number of states near the horizon thus described and the finite entropy of the black hole, which is proportional to the area [13-17]. Though developments in string theory (reviewed in $[6,7]$ ), around the same time, succeeded in resolving the problem, of counting these states and of reproducing the thermodynamical result for the black hole entropy, how to extend this calculation for the case of a "small" number of microstates, i.e. for "small" black holes, remains open $[6,7]$. 
In the next section, we shall introduce an $L / N=a$ ultraviolet — and, at the same time-infrared $L$ cutoff, that will allow us to address this issue by relating this cutoff to the dimension of the Hilbert space of states in the near horizon region.

After this intermezzo, we continue our description of $\mathrm{AdS}_{2}$ as a phase space.

We observe that the variables, $\phi$ and $\mu$, are, in fact, Darboux coordinates with respect to the natural $\mathrm{SO}(2,1)$ invariant Poisson structure, which promotes $\mathrm{AdS}_{2}$ to a phase space. They are conjugate variables and they parametrize the time evolution, at the quantum mechanical level, of the two observers, $O_{a}$ and $O_{b}$, thereby realizing the complementarity of the physics they describe [3].

It is possible also to use the light cone coordinates, $\mu_{ \pm}$to parametrize $\mathrm{AdS}_{2}$, thereby eliminating the angles $\phi_{ \pm}$. The corresponding cosets are:

$$
h\left(\mu_{+}, \mu_{-}\right)=\mathrm{T}_{-}\left(\mu_{-}\right) \mathrm{T}_{+}\left(\mu_{+}\right)
$$

which define a global light cone coordinate system. The map between $\left(\mu_{+}, \mu_{-}\right)$and $\left(x_{0}, x_{1}, x_{2}\right)$ is easily obtained:

$$
\mu_{+}=\frac{x_{1}+x_{2}}{2} \text { and } \mu_{-}=\frac{x_{1}-x_{2}}{1+x_{0}}
$$

The light cone cosets establish the causal patches of any observer on $\mathrm{AdS}_{2}$ and thus the causal diamonds of any pair of observers [48].

For completeness, we exhibit also the standard system of hyperbolic global coordinates,

$$
x_{0}=\cosh \psi \cos \chi, \quad x_{1}=\cosh \psi \sin \chi, \quad x_{2}=\sinh \psi
$$

and the corresponding coset parametrization,

$$
h(\psi, \chi)=\mathrm{R}(\chi) \mathrm{H}(\psi)
$$

with

$$
\mathbf{H}(\psi)=\left(\begin{array}{cc}
\cosh \psi / 2 & \sinh \psi / 2 \\
\sinh \psi / 2 & \cosh \psi / 2
\end{array}\right)
$$

an element of the Lorentz group that acts in the $x_{1}=0$ plane.

These coset parametrizations induce also specific metrics on $\mathrm{AdS}_{2}$. For the parametrization (2.10) we obtain

$$
d s^{2}=\left(1+\mu^{2}\right) d \phi^{2}+2 d \phi d \mu
$$

Substituting in this expression $\mu \equiv \tan \sigma, \sigma \in(-\pi / 2, \pi / 2)$, we obtain the Einstein strip

$$
d s^{2}=\frac{1}{\cos ^{2} \sigma}\left[-d \tau^{2}+d \sigma^{2}\right]
$$

with $\tau \equiv \sigma+\phi \in \mathbb{R}$ with the two disconnected boundaries at $\sigma \equiv \pm \pi / 2$.

For the standard hyperbolic global coordinate system, $(\psi, \chi)$, we obtain the metric

$$
d s^{2}=-\cosh ^{2} \psi d \chi^{2}+d \psi^{2}
$$

with $\psi \in(-\infty, \infty)$ and $\chi \in[0,2 \pi)$. 
Finally, for the light cone coordinates $\mu \equiv \mu_{+}, \nu \equiv \mu_{-}$of eq. (2.14) we find the metric

$$
d s^{2}=-4\left(\nu^{2} d \mu^{2}+\mu^{2} d \nu^{2}-d \mu d \nu\right)
$$

In the rest of this section we discuss the dynamics of probes, appropriate for the description of the string ground state geometry.

The $\mathrm{AdS}_{2}$ coset geometry, inherits a symplectic structure and a non-degenerate Poisson bracket from the isometry group, given by

$$
\left\{x_{0}, x_{1}\right\}=-x_{2} \quad\left\{x_{1}, x_{2}\right\}=x_{0} \quad\left\{x_{2}, x_{0}\right\}=x_{1}
$$

These relations are realized, for example, in the global coordinate system $(\phi, \mu)$, where the area element is $d \phi d \mu$ and the coordinates $\phi$ and $\mu$ are Darboux coordinates, as

$$
\{f, g\}=\frac{\partial f}{\partial \phi} \frac{\partial g}{\partial \mu}-\frac{\partial f}{\partial \mu} \frac{\partial g}{\partial \phi}
$$

The corresponding Hamilton's equations for incompressible flows on $A d S_{2}$ are,

$$
\dot{x}_{\mu}=\left\{x_{\mu}, H\right\}
$$

The simplest, classical, motions of probes of lowest energy, are described by the isometric maps $A \in \mathrm{SL}(2, \mathbb{R})$.

At the level of discrete time evolution (maps), this isometric motion is parametrized as follows: if, for instance, $h(\phi, \mu) \in \mathrm{SL}(2, \mathbb{R})$ is a coset, describing the probe's position in $\mathrm{AdS}_{2}$, at proper time $\tau$, then at time $\tau+1$ it will evolve as

$$
h\left(\phi_{\tau+1}, \mu_{\tau+1}\right)=\mathrm{A} h\left(\phi_{\tau}, \mu_{\tau}\right)(\bmod \mathrm{D})
$$

Using the decompositions (2.7), (2.10), the parameters $\phi_{\mathrm{A}}, \mu_{\mathrm{A}}, \lambda_{\mathrm{A}}$ in can be given explicitly, in terms of the matrix elements of

$$
A=\left(\begin{array}{ll}
a & b \\
c & d
\end{array}\right)
$$

by the expressions

$$
\begin{aligned}
\cos \frac{\phi_{\mathrm{A}}}{2}=\frac{d}{\sqrt{b^{2}+d^{2}}} \quad \sin \frac{\phi_{\mathrm{A}}}{2} & =\frac{b}{\sqrt{b^{2}+d^{2}}} \quad \mu_{\mathrm{A}}=-\frac{a c+b d}{b^{2}+d^{2}} \\
\lambda_{\mathrm{A}} & =\frac{1}{\sqrt{c^{2}+d^{2}}}
\end{aligned}
$$

Applying the above decomposition on the r.h.s. of equation (2.25) we find the l.h.s.

The corresponding Hamiltonians, to the above discrete maps $A \in \operatorname{SL}(2, \mathbb{R})$ must be linear in the generators, $x_{\mu}$, of $\mathrm{SL}(2, \mathbb{R})(2.22)$ and they respect causality.

In our approach the $\mathrm{AdS}_{2}$ radial and time directions are treated as a "phase space" variables, while the evolution time is determined by the group action. Noticing that the stringy uncertainty relations are between the energy and the corresponding physical length, or, in our case, between time and radial extent, the interpretation of $\mathrm{AdS}_{2}$ as a phase space is suitable for strings moving on this background. 
It is essential for the AdS/CFT holographic correspondence to define a conformal compactification of its boundary. The frequently used compactification is the conformal rescaling of the metric giving rise to the Poincare patch, which covers half of the $\mathrm{AdS}_{2}$ spacetime.

Another conformal compactification, in Minkowski signature of $\mathrm{AdS}_{2}$, is obtained by stereographic projection to the $x_{0}=0$ plane. Any point, $\left(\xi_{0}, \xi_{1}, \xi_{2}\right) \in \mathrm{AdS}_{2}$, is projected through the base point $\boldsymbol{p}$ to a point on the plane $x_{0}=0$ with coordinates $\left(x_{1}, x_{2}\right)$ :

$$
\begin{aligned}
& x_{1}=\frac{\xi_{1}}{1-\xi_{0}} \\
& x_{2}=\frac{\xi_{2}}{1-\xi_{0}}
\end{aligned}
$$

Introducing the light cone coordinates of the projection plane, $x_{ \pm} \equiv x_{1} \pm x_{2}$, we can parametrize $\mathrm{AdS}_{2}$ as follows

$$
\begin{aligned}
\xi_{1} & =\frac{x_{+}+x_{-}}{1+x_{+} x_{-}} \\
\xi_{2} & =\frac{x_{+}-x_{-}}{1+x_{+} x_{-}} \\
\xi_{0} & =\frac{x_{+} x_{-}-1}{1+x_{+} x_{-}}
\end{aligned}
$$

We observe that the stereographic projection from the point $\boldsymbol{p}=(1,0,0)$, maps each of the light cones, $\boldsymbol{l}_{ \pm}(\mathbf{p})=\{(1, \pm \mu, \mu) \mid \mu \in \mathbb{R}\}$, to two points on the boundaries.

In order to parametrize uniquely the points on $\boldsymbol{l}_{ \pm}(\boldsymbol{p})$, we must use the stereographic projection from the "antipode", $\boldsymbol{q}=(-1,0,0)$. If we call the new coordinates on the $x_{0}=0$ plane, $y_{ \pm}$, we have

$$
\begin{aligned}
\xi_{1} & =\frac{y_{+}+y_{-}}{1+y_{+} y_{-}} \\
\xi_{2} & =\frac{y_{+}-y_{-}}{1+y_{+} y_{-}} \\
\xi_{0} & =\frac{1-y_{+} y_{-}}{1+y_{+} y_{-}}
\end{aligned}
$$

This coordinate system has the same problem for the points on the light cones $\boldsymbol{l}_{ \pm}(\boldsymbol{q})$. We easily check that the stereographic projection from $\boldsymbol{p}$ (resp. $\boldsymbol{q}$ ) maps the light cone axes, $x_{+}=0$ or $x_{-}=0$ of the projective plane, $x_{0}=0$ to $\boldsymbol{l}_{ \pm}(\boldsymbol{q})$ (resp. $\boldsymbol{l}_{ \pm}(\boldsymbol{p})$ ). More generally, the curves, on $\mathrm{AdS}_{2}$, defined by $x_{+}=$const or $x_{-}=$const (correspondingly for $y_{ \pm}$) are the light-cone straight lines, which generate $\mathrm{AdS}_{2}$.

In the overlap region, of the two coordinate systems, the transition functions are

$$
x_{-} y_{+}=1=x_{+} y_{-}
$$

The induced metric, in terms of $x_{ \pm}$takes the form:

$$
d s^{2}=4 \frac{d x_{+} d x_{-}}{\left(1+x_{+} x_{-}\right)^{2}}
$$

and, similarly, for $y_{ \pm}$. 
We observe now that the induced metric is invariant under the Möbius transformations

$$
\begin{aligned}
& x_{+} \rightarrow \mathrm{A} x_{+} \equiv \frac{a x_{+}+b}{c x_{+}+d} \\
& x_{-} \rightarrow\left[\mathrm{A}^{-1}\right]^{\mathrm{T}} x_{-} \equiv \frac{d x_{-}-c}{-b x_{-}+a}
\end{aligned}
$$

These transformations result from the Weyl action (2.4) as can be checked using the stereographic light cone parametrizations of $\mathrm{AdS}_{2}$ (2.29) and (2.30). We remark that the variables $\left(x_{+}, x_{-}\right)$do not mix under the isometry group, in contrast to the other coordinate systems.

By definition the following identity holds, for any $A \in S L(2, \mathbb{R})$ :

$$
\left[\mathrm{A}^{-1}\right]^{\mathrm{T}}=\varepsilon \mathrm{A} \varepsilon^{\mathrm{T}}
$$

where

$$
\varepsilon \equiv\left(\begin{array}{cc}
0 & 1 \\
-1 & 0
\end{array}\right)
$$

Therefore, eq. (2.33) implies that $\left(x_{+}, x_{-}\right)$are conjugate variables and the stereographic projection plane is promoted to a phase space. Indeed, the $\mathrm{AdS}_{2} / \mathrm{CFT}_{1}$ correspondence is based on the fact that $\mathrm{SL}(2, \mathbb{R})$ has three different incarnations: (a) as the isometry group of $\mathrm{AdS}_{2}$, (b) as the symplectic group of $\mathrm{AdS}_{2}$, as a phase space and (c) as the conformal, Möbius, group of the boundary $\mathrm{CFT}_{1}$.

The variables, $x_{ \pm}$, are thus appropriate holographic variables, because the isometry transformation group of $\mathrm{AdS}_{2}$ is reduced on them to two, conjugated, copies, of the $1 \mathrm{~d}$ Möbius conformal group.

We come now to the parametrization of the boundary, which is disconnected and consists of two circles at $x_{2} \rightarrow \pm \infty$. In the covering space the boundary is $\mathbb{R} \times\{1,-1\}$.

Because of their transformation properties, the variables $x_{ \pm}$are the most suitable to use to define the two disconnected components of the boundary, by the branches of the hyperbola (cf. eq. (2.29))

$$
1+x_{+} x_{-}=0
$$

This relation allows us to write $x_{+}\left(\right.$resp. $\left.x_{-}\right)$as a Möbius transformation of the other:

$$
x_{+}=-\frac{1}{x_{-}} \equiv \varepsilon \cdot x_{-}
$$

This relation is invariant under the Möbius transformations (2.33). The two components of the boundary are, therefore, two copies of the projective line, $\mathbb{R P}^{1}$.

We notice here that the stereographic projection maps each one of the boundary components to the two branches of the hyperbola.

The boundary can also be described as the coset space, $\operatorname{SL}(2, \mathbb{R}) / \mathfrak{B}$, where $\mathfrak{B}$ is the Borel subgroup of dilatations and translations,

$$
\mathfrak{B}=\left\{\mathrm{B}(b, \lambda)=\left(\begin{array}{cc}
\lambda & b \\
0 & \lambda^{-1}
\end{array}\right) \mid \lambda \in \mathbb{R}^{*}, b \in \mathbb{R}\right\}
$$

which preserves the point at infinity, $\left(x_{+}=\infty, x_{-}=0\right)$. 
For any $\mathrm{A} \in \mathrm{SL}(2, \mathbb{R})$ we have the decomposition

$$
\mathrm{A}=\mathrm{R}(\phi) \mathrm{B}(b, \lambda)
$$

and the elements $\mathrm{R}(\phi) \in \mathrm{SO}(2, \mathbb{R})$ parametrize the boundary.

It will be useful later to parametrize the bulk coset representatives, $h(\phi, \mu)$ and the boundary representatives, $\mathrm{R}(\phi)$ by the light cone coordinates $x_{ \pm}$. The map is the following:

$$
\begin{aligned}
& x_{+}=\frac{1}{\tan \frac{\phi}{2}} \\
& x_{-}=\frac{1-\mu \tan \frac{\phi}{2}}{\mu+\tan \frac{\phi}{2}}
\end{aligned}
$$

The boundary is reached when $\mu \rightarrow \pm \infty$. Indeed, a measure of the distance from the boundary is

$$
z \equiv 1+x_{+} x_{-}=\frac{2}{\sin \phi\left(\mu+\tan \frac{\phi}{2}\right)}
$$

The coset representatives of the bulk and the boundary become functions $h\left(x_{+}, x_{-}\right)$and $\mathrm{R}\left(x_{+}\right)$respectively. So $x_{+}$parametrizes motions parallel to the boundary and $x_{-}$motions towards the boundary.

To relate the classical action of $\mathrm{SL}(2, \mathbb{R})$ in the bulk $(2.25)$ with the corresponding action on the boundary, defined as

$$
\mathrm{R}\left(\phi_{\tau+1}\right)=\operatorname{AR}\left(\phi_{\tau}\right)
$$

we must compute the r.h.s. through the decomposition

$$
\operatorname{AR}\left(\phi_{\tau}\right)=\mathrm{R}\left(\phi_{\tau+1}\right) \mathrm{B}\left(b_{\tau+1}, \lambda_{\tau+1}\right)
$$

and mod out the Borel factor $\mathrm{B}\left(b_{\tau+1}, \lambda_{\tau+1}\right)$.

Closing this section we explain in the following, why we have chosen to parametrize the bulk - boundary geometry and dynamics by the corresponding group cosets. The basic reason is their role in a new proposal for the holographic correspondence,which we think presents an extension of the standard $\mathrm{AdS}_{2} / \mathrm{CFT}_{1}$ one.

The $\mathrm{R}(\phi)$ coset representatives, by construction, cannot detect the distance from the boundary, i.e. $x_{-}$. Only at the quantum level, where an uncertainty relation, between $x_{+}$ and $x_{-}$, exists and it reflects the UV/IR connection, is it possible from the distribution of $x_{+}$on the boundary to get information for the distribution of $x_{-}$in the bulk.

The quantum mechanical states on the boundary and those in the bulk, which maximize the flow of quantum mechanical information between the bulk and the boundary, given the coset structure of their geometries, are the corresponding coherent states (wavelets).

They form overcomplete sets of states with classical transformation properties but they are powerful enough to describe quantum dynamics and geometry at the same time [49]. We shall present the construction of these states and their properties in the next section, after we have introduced the modular discretization of the geometry and dynamics on $\mathrm{AdS}_{2}$. 


\section{Modular discretization and quantum dynamics on $\operatorname{AdS}_{2}[N]$}

Recent discussions on the quantum black hole entropy of extremal black holes and the $\mathrm{AdS}_{2} / \mathrm{CFT}_{1}$ correspondence suggest the identification of the black hole entropy with the logarithm of the string ground state degeneracy [8-10]. This is an integer, $N$, fixed by the set of the black hole's electric and magnetic charges.

Since in the Hilbert space of the degenerate ground state, we have at most $N$ linearly independent wave functions, the geometry resolved by the probe is fuzzy, with resolution $1 / N$.

In order to model the geometry and the dynamics of black hole information processing, we should take into account the following constraints, which have been discussed in the literature on the black hole information paradox:

- In the vicinity of the black hole horizon, the dynamics is chaotic and strongly mixing. Any additional bit of information, that falls into the black hole, in a very short time, reaches (dynamic) equilibrium with the other microscopic degrees of freedom comprising the blackhole horizon.

Furthermore, the mixing should be holographic: any subset of horizon qubits has a coarse-grained representation of the total infalling information.

This leads to the following constraints on the geometry and the dynamics:

- Randomness, non-locality and factorization of the space-time geometry, which implies that the total Hilbert space factorizes into a tensor product of local, coarse-grained, Hilbert spaces [50-53].

- The dynamics should provide the fastest quantum information processing, saturating the scrambling time bound [54-58].

We propose to model this random, non-local and factorizable geometry by a numbertheoretic discretization, that preserves the corresponding group-theoretical structure of $\mathrm{AdS}_{2}$ spacetime. This is done by replacing $\mathrm{AdS}_{2}$ by the discrete cosets, $\operatorname{AdS}_{2}[N]=$ $\mathrm{SL}\left(2, \mathbb{Z}_{N}\right) / \mathrm{SO}\left(1,1, \mathbb{Z}_{N}\right)$. We thereby replace the set of real numbers, $\mathbb{R}$, by the set of integers modulo $N$. We call this "modular discretization". This is a finite, random, set of points in the embedding Minkowski spacetime $\mathscr{M}^{2,1}$. In the mathematical literature, such a set of points is called a finite geometry [24, 25]. Introducing appropriate length scales and taking the large $N$ limit we can check that the smooth geometry of $\mathrm{AdS}_{2}$ emerges.

To accommodate the above requirements on the dynamics, we employ discrete time maps. These are the Arnol'd cat maps, $A$ in $\mathrm{SL}\left(2, \mathbb{Z}_{N}\right)$. These are known to exhibit strong mixing, ergodic properties [26-28,34,35]., non-locality and factorization in the cutoff discretization parameter, $N[29,38]$.

We restrict our construction to the case $N=p$ prime for the technical simplicity of the presentation of our arguments. In this case, the set of integers modulo $p$ is the simplest Galois field, $\mathbb{F}_{p}$. The unitary, irreducible, representations of the isometry group of $\operatorname{AdS}_{2}[p]$, $\mathrm{SL}\left(2, \mathbb{F}_{p}\right)$, are known [45]. 
The restriction to $N$ prime can be removed by noticing some interesting factorizations: if $N=N_{1} N_{2}$, with $N_{1,2}$ coprime, then we have [29]

$$
\mathrm{SL}\left(2, \mathbb{Z}_{N_{1} N_{2}}\right)=\mathrm{SL}\left(2, \mathbb{Z}_{N_{1}}\right) \otimes \mathrm{SL}\left(2, \mathbb{Z}_{N_{2}}\right)
$$

and

$$
\operatorname{AdS}_{2}\left[N_{1} N_{2}\right]=\operatorname{AdS}_{2}\left[N_{1}\right] \otimes \operatorname{AdS}_{2}\left[N_{2}\right]
$$

These factorizations imply that all powers of primes, $2^{n_{1}}, 3^{n_{2}}, 5^{n_{3}}, \ldots$, are the building blocks of our construction. The physical interpretation of this factorization is that the most coarse-grained Hilbert spaces on the horizon have dimensions powers of primes.

We observe that taking tensor products over all powers of a fixed prime, $p$, we can model dynamics over the $p$-adic spacetime, $\operatorname{AdS}_{2}\left[\mathbb{Q}_{p}\right]$.

In order to study the finite geometry of $\mathrm{AdS}_{2}[p]$, we recall the following facts about its "isometry group", $\mathrm{SL}\left(2, \mathbb{F}_{p}\right)$ :

The order of $\operatorname{SL}\left(2, \mathbb{F}_{p}\right)$ is $p\left(p^{2}-1\right)$. For the subgroups of rotations, $\mathrm{R}$, translations, $\mathrm{T}_{ \pm}$ and dilatations, $\mathrm{D}$, the orders are $p+1, p$ and $p-1$ respectively. So the finite geometry of $\operatorname{AdS}_{2}[p]$ has $p(p+1)$ points.

The set of points of the finite geometry of $\mathrm{AdS}_{2}[p]$ is, by definition, the set of all solutions of the equation

$$
x_{0}^{2}+x_{1}^{2}-x_{2}^{2} \equiv 1 \bmod p
$$

This can be parametrized as follows:

$$
\begin{aligned}
& x_{0} \equiv(a-b \mu) \bmod p \\
& x_{1} \equiv(b+a \mu) \bmod p \\
& x_{2} \equiv \mu \bmod p
\end{aligned}
$$

where $a^{2}+b^{2} \equiv 1 \bmod p$ and $a, b, \mu \in \mathbb{F}_{p}$.

The points of $\mathrm{AdS}_{2}[p]$ comprise the bulk-we must add the points on the boundary.

The boundary is the "mod $p$ " projective line, $\mathbb{R P}_{p}^{1}$, defined as the set

$$
\mathbb{R P}_{p}^{1}=G F^{*}[p] \cup\{0, \infty\}
$$

so the number of boundary points (cosets) is $p+1$.

We shall now define the quantum mechanics of the probes of the bulk $\operatorname{AdS}_{2}[p]$ and its boundary, as well as the corresponding coherent states [49].

We start with the construction of finite quantum mechanics (FQM) in the bulk. It is obvious that the set of the states and the set of observables should carry a representation of the coset structure of the bulk. We choose the space of states to be the Hilbert space, of dimension $p$, of the metaplectic representation of $\operatorname{SL}\left(2, \mathbb{F}_{p}\right)$ [37]. This choice is motivated by the fact that the spatial part of $\mathrm{AdS}_{2}[p]$ is the finite field $\mathbb{F}_{p}$, the set of values of the space-like variable $x_{-}$. The wavefunctions will be the normalized elements of the complex, projective, space $\mathbb{C P}^{p-1}$.

In the papers $[31,37,38]$ the explicit construction of the metaplectic representation of $\mathrm{SL}\left(2, \mathbb{F}_{p}\right)$ has been presented,as well as various sets of coherent states. 
The building blocks of the observables of FQM are two $p \times p$, unitary, matrices, "clock", $Q$ and "shift", $P$, representing the "exponentials" of the position and momentum operators (for periodic boundary conditions) [59-62]:

$$
Q_{k, l}=\omega^{k} \delta_{k, l}, \quad P_{k, l}=\delta_{k-1, l},
$$

$k, l \in \mathbb{F}_{p}$ and $\omega=\exp (2 \pi \mathrm{i} / p)$ is the $p$ th root of unity.

These matrices satisfy the exponentiated Heisenberg-Weyl commutation relation

$$
Q P=\omega P Q
$$

A useful basis is provided by the magnetic translations

$$
J_{r, s}=\omega^{r \cdot s / 2} P^{r} Q^{s},
$$

elements of the (finite) Heisenberg-Weyl group, where the $1 / 2$ in the exponent is computed $\bmod p$.

The $J_{r, s}$ realize a projective representation of the translation group on the discrete torus, $\mathbb{T}_{p}=\mathbb{F}_{p} \times \mathbb{F}_{p}$ :

$$
J_{r, s} J_{r^{\prime}, s^{\prime}}=\omega^{\left(r^{\prime} s-r s\right) / 2} J_{r+r^{\prime}, s+s^{\prime}}
$$

are unitary

$$
\left[J_{r, s}\right]^{\dagger}=J_{-r,-s}
$$

and periodic

$$
\left[J_{r, s}\right]^{p}=I_{p \times p}
$$

The phase factor in eq. (3.9) is a cocycle and represents the non-commutativity of the quantized torus, $\mathbb{T}_{\theta}(\theta=2 \pi / p)[63,64]$.

The exact quantization of Arnol'd cat maps, $A \in \mathrm{SL}\left(2, \mathbb{F}_{p}\right)$, is given by unitary matrices, $U(A)$, satisfying

$$
\mathrm{U}(\mathrm{A}) J_{r, s} \mathrm{U}(\mathrm{A})^{\dagger}=J_{(r, s) \mathrm{A}^{-1}}
$$

This is the definition of the metaplectic representation of $\operatorname{SL}\left(2, \mathbb{F}_{p}\right)$, which, in general, is projective.

We can find a proper representation of $\operatorname{SL}\left(2, \mathbb{F}_{p}\right)$ which, then satisfies the relation

$$
\mathrm{U}(\mathrm{A}) \mathrm{U}(\mathrm{B})=\mathrm{U}(\mathrm{AB})
$$

for all $\mathrm{A}, \mathrm{B} \in \mathrm{SL}\left(2, \mathbb{F}_{p}\right)$. This can be done because of the following theorem: every projective representation of $\operatorname{SL}\left(2, \mathbb{F}_{p}\right)$ can be lifted to a proper representation [65].

The proper representation that corresponds to the metaplectic one is given by the following expression $[36,37]$

$$
[\mathrm{U}(\mathrm{A})]_{k, l}=\frac{1}{\sqrt{p}}(-2 c \mid p)\left\{\begin{array}{c}
1 \\
-\mathrm{i}
\end{array}\right\} \omega^{-\frac{a k^{2}-2 k l+d l^{2}}{2 c}}
$$


for $c \not \equiv 0 \bmod p$ and the Jacobi symbol, $(-2 c \mid p)= \pm 1$, depending on whether $-2 c$ is a quadratic residue mod $p$ or not and the upper term between the brackets pertains if $p=4 k+1$, while the lower if $p=4 k-1$.

In the case $c \equiv 0 \bmod p$ and $a \in \mathbb{F}_{p}^{*}$, then

$$
A=\left(\begin{array}{cc}
a & b \\
0 & a^{-1}
\end{array}\right)
$$

and

$$
\mathrm{U}(\mathrm{A})_{k, l}=\frac{(-a \mid p)}{\sqrt{p}}\left\{\begin{array}{c}
1 \\
-1
\end{array}\right\} \omega_{p}^{-\frac{a b}{2} k^{2}} \delta_{k, a^{-1} l}
$$

An important application of eq. (3.14) is for the Quantum Fourier Transform (QFT). For

$$
\mathrm{F}=\left(\begin{array}{cc}
0 & -1 \\
1 & 0
\end{array}\right)
$$

the corresponding unitary operator is given by

$$
\mathrm{U}(\mathrm{F})=\frac{1}{\sqrt{p}}(-2 \mid p)\left\{\begin{array}{c}
1 \\
-\mathrm{i}
\end{array}\right\} \omega_{p}^{k l}=(-2 \mid p)\left\{\begin{array}{c}
1 \\
-\mathrm{i}
\end{array}\right\} F
$$

and

$$
F_{k, l}=\frac{1}{\sqrt{p}} \omega_{p}^{k l}
$$

is the QFT matrix.

The representation given in eq. (3.14) is reducible: it is decomposed into two, irreducible, components [36]:

$$
\mathrm{U}(\mathrm{A})_{\mathrm{L}, \mathrm{R}}=\mathrm{U}(\mathrm{A}) \frac{I_{p \times p} \pm S}{2}
$$

where

$$
S=F^{2}
$$

From eqs. (3.17) and (3.18) we deduce that

$$
\mathrm{U}\left(\mathrm{F}^{4}\right)=F^{4}=I_{p \times p}
$$

and, thus, the eigenvalues of $S$ are \pm 1 , which label the chiralities of the two irreducible components. The dimension of the corresponding eigenspaces is $(p \pm 1) / 2$.

It is possible to generalize the metaplectic representation from the discretization $N=p$ prime to any integer $N$ by noting that, if $N$ is composite, $N=N_{1} N_{2}$, with $N_{1}, N_{2}$ coprime, for every $\mathrm{A} \in \mathrm{SL}\left(2, \mathbb{Z}_{N_{1} N_{2}}\right)$ we obtain

$$
A=A_{1} \cdot A_{2}
$$

with $\mathrm{A}_{i} \in \mathrm{SL}\left(2, \mathbb{Z}_{N_{i}}\right), i=1,2$. 
It can be proved that the unitary matrix $\mathrm{U}(\mathrm{A})$ of eq. (3.14) "inherits" this property as follows

$$
\mathrm{U}(\mathrm{A})=\mathrm{U}\left(\mathrm{A}_{1}\right) \otimes \mathrm{U}\left(\mathrm{A}_{2}\right)
$$

The $N_{1} N_{2} \times N_{1} N_{2}$ matrix $\mathrm{U}(\mathrm{A})$ decomposes into a tensor product of an $N_{1} \times N_{1}$ and an $N_{2} \times N_{2}$ unitary matrix. This leads to an acceleration of the computation of the action of the quantum map $\mathrm{U}(\mathrm{A})$ on the Hilbert space of states, $\mathcal{H}_{N_{1} N_{2}}$, from $O\left(N^{2}\right)$ to $O(N \ln N)$ operations [29].

Thus, the building blocks of FQM are the Hilbert spaces of dimension $N=p^{n}$, with $p$ an odd prime and $n \in \mathbb{N}$.

\section{The coherent states of bulk and boundary}

The coherent state method selects a state, invariant under the stability group, as the ground state, $|0\rangle_{\mathrm{D}}[66,67]$.

For the bulk the stability group is the scaling group, D. The corresponding quantum map, $\mathrm{U}(\mathrm{D}(\lambda))$, for $\lambda \in \mathbb{F}_{p}^{*}$, is the circulant matrix:

$$
\mathrm{U}(\mathrm{D}(\lambda))_{k, l}=(-\lambda \mid p)\left\{\begin{array}{c}
1 \\
-1
\end{array}\right\} \delta_{k, \lambda^{-1} l}
$$

We choose as ground state a common eigenvector of $\mathrm{U}(\mathrm{D}(\lambda))$ for all $\lambda$, namely

$$
|0\rangle_{\mathrm{D}}=\frac{1}{\sqrt{p}}(\underbrace{1,1, \ldots, 1}_{p})
$$

The coherent states for $\mathrm{AdS}_{2}[p]$ are now defined as

$$
|h\rangle=\mathrm{U}(\mathrm{h})|0\rangle_{\mathrm{D}}
$$

for all $h \in \operatorname{AdS}_{2}[p]$ and $\mathrm{U}(h)$ the $p \times p$ unitary matrix constructed in eq. (3.16).

We notice here that the vacuum, $|0\rangle_{\mathrm{D}}$, is annihilated by the projector $P_{-}=(I-S) / 2$, so it belongs to the subspace of the projector, $P_{+}=(I+S) / 2$, which has dimension $p_{+} \equiv$ $(p+1) / 2$. The matrix $S$ commutes with all matrices $\mathrm{U}(\mathrm{h})$ and this implies that the coherent states, $|h\rangle$, also, belong to the eigenspace of $P_{+}$. This is the positive chirality eigenspace. It is possible to construct coherent states, belonging to the orthogonal eigenspace of dimension $p_{-}=(p-1) / 2$, by choosing the common eigenstate of the dilatation group among the eigenvectors of $S$ with opposite chirality.

We can use the parametrization of the cosets by rotations and translations in order to obtain explicit expressions for the coherent states, $|h\rangle$ :

For

$$
h(\phi, \mu)=\left(\begin{array}{cc}
a & -b \\
b & a
\end{array}\right)\left(\begin{array}{cc}
1 & -\mu \\
0 & 1
\end{array}\right)
$$


with $a^{2}+b^{2} \equiv 1 \bmod p$. Using eqs. (2.40) we find the relation between $a, b, \mu$ and $x_{ \pm}$, namely

$$
\begin{aligned}
& x_{+}=\frac{a}{b} \\
& x_{-}=\frac{a-b \mu}{a \mu+b}
\end{aligned}
$$

In components:

$$
\left.\left.\langle k \mid h\rangle=\frac{1}{\sqrt{p}}((a-b \mu)) \mid p\right) \omega_{p}^{\frac{b+\mu a}{2(a-b \mu)} k^{2}}=\frac{1}{\sqrt{p}}((a-b \mu)) \mid p\right) \omega_{p}^{\frac{k^{2}}{2 x-}}
$$

where $k=0,1,2, \ldots, p-1$. The coherent states, $|h\rangle$, of the bulk, can be parametrized therefore in terms of $x_{ \pm}$and will be denoted $\left|x_{+}, x_{-}\right\rangle$.

These definitions imply the classical transformation of coherent states under the isometry group, namely

$$
\mathrm{U}(\mathrm{A})|h\rangle=|\mathrm{A} h\rangle
$$

These states form an overcomplete set of normalized states with very useful properties:

- Resolution of the identity:

$$
\frac{1}{d} \sum_{h}|h\rangle\langle h|=P_{+}
$$

where $d$ is defined

$$
d=\sum_{h}\left|\left\langle h \mid h_{1}\right\rangle\right|^{2}
$$

for any state $\left|h_{1}\right\rangle$.

The above identity is based on the irreducibility of the metaplectic representation on the subspace of positive chirality.

- Propagator property for the function

$$
\Delta\left(h_{1}, h_{2}\right)=\left\langle h_{2} \mid h_{1}\right\rangle
$$

This function has the property of a "reproducing kernel" (propagator)

$$
\Delta\left(h_{1}, h_{2}\right)=\frac{1}{d} \sum_{h} \Delta\left(h_{1}, h\right) \Delta\left(h, h_{2}\right)
$$

and is invariant under the isometry group.

- For a general state $|\psi\rangle$ we have

$$
|\psi\rangle=\frac{1}{d} \sum_{h}|h\rangle\langle h \mid \psi\rangle
$$


- The symbol of operators: to an operator $\widehat{A}$ we associate a scalar function $\widetilde{A}\left(h_{1}, h_{2}\right)$ with

$$
\widetilde{A}\left(h_{1}, h_{2}\right) \equiv\left\langle h_{2}|\widehat{A}| h_{1}\right\rangle
$$

so that

$$
\widehat{A}=\frac{1}{d^{2}} \sum_{h_{1}, h_{2}} \widetilde{A}\left(h_{1}, h_{2}\right)\left|h_{2}\right\rangle\left\langle h_{1}\right|
$$

For two operators $\widehat{A}, \widehat{B}$ we assign as symbol of their product the expression

$$
\widetilde{A B}\left(h_{1}, h_{2}\right)=\frac{1}{d} \sum_{h} \widetilde{A}\left(h_{1}, h\right) \widetilde{B}\left(h, h_{2}\right)
$$

The local quantum observables, that have "nice" transformation properties, are the $p \times p$ hermitian matrices $Q_{I}(h)$, such that

$$
\mathrm{U}(\mathrm{A}) Q_{I}(h) \mathrm{U}(\mathrm{A})^{\dagger}=R_{I J} Q_{J}(\mathrm{~A} h), I, J=1, \ldots, \operatorname{dim} R
$$

For scalar observables (scalar fields) we must have

$$
\mathrm{U}(\mathrm{A}) Q(h) \mathrm{U}(\mathrm{A})^{\dagger}=Q(\mathrm{~A} h)
$$

The simplest ones are the (pure state) density matrices,

$$
\rho(h)=|h\rangle\langle h|
$$

that can be used as a basis for measurement.

For any scalar function, $f(h)$, on $\operatorname{AdS}_{2}[p]$, we can construct the quantum observable

$$
\mathcal{O}(f) \equiv \sum_{h} f(h)|h\rangle\langle h|
$$

which is hermitian if $f(\cdot)$ is real.

The one-time-step evolution of these observables is given by

$$
\mathcal{O}_{n+1}(f)=\mathrm{U}(\mathrm{A}) \mathcal{O}_{n}(f) \mathrm{U}(\mathrm{A})^{\dagger}
$$

with initial condition $\mathcal{O}_{n=0}(f)=\mathcal{O}(f)$.

We may write this relation in the following way:

$$
\mathcal{O}_{n+1}(f)=\mathcal{O}_{n}\left(f \circ \mathrm{A}^{-1}\right)
$$

The set of time correlation functions for these observables defines FQM on $\operatorname{AdS}_{2}[p]$ :

$$
G\left(t_{1}, t_{2}, \ldots, t_{n} \mid f_{1}, f_{2}, \ldots, f_{n}\right)={ }_{\mathrm{D}}\left\langle 0\left|\mathcal{O}_{t_{1}}\left(f_{1}\right) \mathcal{O}_{t_{2}}\left(f_{2}\right) \ldots \mathcal{O}_{t_{n}}\left(f_{n}\right)\right| 0\right\rangle_{\mathrm{D}}
$$

We shall present the bulk/boundary correspondence for the quantum map dynamics using the parametrization of both spaces by the light cone variables, $x_{ \pm}$, of the stereographic 
projection. The coherent state $|h\rangle \equiv\left|x_{+}, x_{-}\right\rangle$and the action of an $\operatorname{SL}\left(2, \mathbb{F}_{p}\right)$ group element A will be lifted to the action of the unitary operator, $U(A)$ as follows:

$$
\mathrm{U}(\mathrm{A})\left|x_{+}, x_{-}\right\rangle=\left|\frac{a x_{+}+b}{c x_{+}+d}, \frac{d x_{-}-c}{a-b x_{-}}\right\rangle
$$

Let us now pass to the construction of the coherent states and the observables on the boundary.

The boundary, $\operatorname{Bd}[p]$, of the discrete space-time, $\operatorname{AdS}_{2}[p]$, is defined, in analogy with the continuum case, by conformal compactification. In light-cone coordinates, $\left(x_{+}, x_{-}\right)$, of the projective plane, it is described as the set of points

$$
1+x_{+} x_{-} \equiv 0 \bmod p
$$

For every $x_{+} \in \mathbb{F}_{p}^{*}$, we have $x_{-} \equiv-x_{+}^{-1} \bmod p$. We must, also, add the points at "infinity", $x_{+}=0, x_{-}=\infty$ and $x_{+}=\infty, x_{-}=0$. So the boundary comprises the $p+1$ points

$$
\mathrm{Bd}[p]=\left\{\left(x_{+},-x_{+}^{-1}\right) \mid x_{+} \in \mathbb{F}_{p}^{*}\right\} \cup\{(\infty, 0),(0, \infty)\}
$$

The boundary set is invariant under the Möbius group, $\operatorname{SL}\left(2, \mathbb{F}_{p}\right)$ and is the coset space $\operatorname{SL}\left(2, \mathbb{F}_{p}\right) / \mathfrak{B}_{p}$, where $\mathfrak{B}_{p}$ is the Borel subgroup, that preserves the "point at infinity", $q=\left(x_{+}=\infty, x_{-}=0\right)$ :

$$
\mathfrak{B}_{p}=\left\{\left(\begin{array}{cc}
\lambda & b \\
0 & \lambda^{-1}
\end{array}\right) \mid \lambda \in \mathbb{F}_{p}^{*}, b \in \mathbb{F}_{p}\right\}
$$

In the $p$-dimensional Hilbert space of the metaplectic representation the quantum maps, corresponding to $\mathfrak{B}_{p}$, are given as

$$
U\left[\left(\begin{array}{cc}
\lambda & b \\
0 & \lambda^{-1}
\end{array}\right)\right]_{k, l}=(-\lambda \mid p)\left\{\begin{array}{c}
1 \\
-1
\end{array}\right\} \omega_{p}^{-\frac{\lambda b}{2} k^{2}} \delta_{k, \lambda^{-1} l}
$$

The "vacuum" on which we define the coherent states of the boundary must be a common eigenvector of the stability group $\mathfrak{B}_{p}[49]$. We can check from (4.27) that there is only one such eigenvector, $|0\rangle_{q}$ :

$$
\langle k \mid 0\rangle_{q}=\delta_{k, 0}
$$

The subgroup of $\operatorname{SL}\left(2, \mathbb{F}_{p}\right)$, that acts transitively on the boundary is generated by the rotation subgroup $\mathrm{SO}\left(2, \mathbb{F}_{p}\right)$ :

$$
\mathrm{SO}\left(2, \mathbb{F}_{p}\right)=\left\{\left(\begin{array}{cc}
a & -b \\
b & a
\end{array}\right) \mid a^{2}+b^{2} \equiv 1 \bmod p\right\}
$$

This is an abelian, cyclic, subgroup of order $p+1$, with generator that can be found by random search [37].

The discrete coherent states of the boundary $\mathrm{Bd}[p]$ can now be defined as

$$
\left|x_{+}\right\rangle=\mathrm{U}\left(\mathrm{R}\left(x_{+}\right)\right)|0\rangle_{q}
$$


From (3.14) we obtain the expression for the ground state wave function, $\left\langle k \mid x_{+}\right\rangle$as

$$
\left\langle k \mid x_{+}\right\rangle=\frac{1}{\sqrt{p}}(-2 b \mid p)\left\{\begin{array}{l}
1 \\
\mathrm{i}
\end{array}\right\} \omega_{p}^{-\frac{x_{+} k^{2}}{2}}
$$

when $x_{+} \in \mathbb{F}_{p}^{*}$. For the two additional points of the boundary, $x_{+}=\infty, x_{-}=0($ then $b=0)$ and $x_{+}=0, x_{-}=\infty$ (then $a=0$ ) the corresponding coherent states are:

- When $b=0$, we need, in principle, to distinguish two cases: $a=+1$ and $a=-1$ - but, since the action of the group is projective, these lead to the same state, $\left|x_{+}=\infty\right\rangle=|0\rangle_{q}$, whence $\left\langle k \mid x_{+}\right\rangle=\delta_{k, 0}$.

- For $a=0$, then $b=-1, x_{+}=0$ The state $\left|x_{+}=0\right\rangle$ leads to the constant wavefunction

$$
\left\langle k \mid x_{+}\right\rangle=\frac{1}{\sqrt{p}}(2 \mid p)\left\{\begin{array}{l}
1 \\
\mathrm{i}
\end{array}\right\}
$$

for all $k=0,1, \ldots, p-1$.

In total we get $p+1$ states, matching the number of points on the boundary.

In analogy with the bulk coherent states, we observe, that the ground state, $|0\rangle_{q}$, of the boundary, is annihilated by the projector $(I-S) / 2$, as are all the coherent states, $\left|x_{+}\right\rangle$. Thus, these coherent states live in the eigenspace of $P_{+}=(I+S) / 2$, which is $(p+1) / 2-$ dimensional. They form an overcomplete set of states and display all the expected features of coherent states.

Let us now turn our attention to the boundary observables. We construct the following class of operators, which have nice transformation properties under the Möbius conformal group and form a basis for measurement.

Using the magnetic translations, $J_{r, s}$, of the Heisenberg-Weyl group, where $r, s=$ $0,1,2, \ldots, p-1$, we define the operators

$$
\mathcal{O}\left(x_{+}\right)=\frac{1}{p} \sum_{s=0}^{p-1} J_{s\left(1,-x_{+}\right)}
$$

with $x_{+}=0,1, \ldots, p-1$. Their matrix elements are

$$
[\mathcal{O}]_{k, l}=\frac{1}{p} \omega_{p}^{-\frac{x_{+}\left(k^{2}-l^{2}\right)}{2}}
$$

These operators are projectors:

$$
\begin{aligned}
& \mathcal{O}\left(x_{+}\right)^{2}=\mathcal{O}\left(x_{+}\right) \\
& \mathcal{O}\left(x_{+}\right)^{\dagger}=\mathcal{O}\left(x_{+}\right)
\end{aligned}
$$

and they transform conformally (for all $\mathrm{A} \in \mathrm{SL}\left(2, \mathbb{Z}_{N}\right)$ ):

$$
\mathrm{U}(\mathrm{A}) \mathcal{O}\left(x_{+}\right) \mathrm{U}(\mathrm{A})^{\dagger}=\mathcal{O}\left(\frac{a x_{+}+b}{c x_{+}+d}\right)
$$


For example, under the Fourier transform,

$$
\mathrm{S}=\left(\begin{array}{cc}
0 & -1 \\
1 & 0
\end{array}\right)
$$

we find that

$$
\mathrm{U}(\mathrm{S}) \mathcal{O}\left(x_{+}\right) \mathrm{U}(\mathrm{S})^{\dagger}=\mathcal{O}\left(-\frac{1}{x_{+}}\right)
$$

We use eq. (4.37) to define

$$
\mathcal{O}\left(x_{+}=\infty\right) \equiv \mathrm{U}(\mathrm{S}) \mathcal{O}(0) \mathrm{U}(\mathrm{S})^{\dagger}=|0\rangle_{q q}\langle 0|
$$

In the notation of eq. (4.30) the state $|\infty\rangle$ is the ground state, $|0\rangle_{q}$.

The operators $\mathcal{O}\left(x_{+}\right)$have the nice property that they are projectors on the discrete coherent states, $\left|x_{+}\right\rangle$. One can, indeed, check that

$$
\mathcal{O}\left(x_{+}\right)=\left|x_{+}\right\rangle\left\langle x_{+}\right|
$$

This holds for all $x_{+}=0,1,2, \ldots, p-1, \infty$.

The boundary observables can, therefore, be expressed in the $\mathcal{O}\left(x_{+}\right)$basis: to any function, $f: \operatorname{Bd}[N] \rightarrow \mathbb{C}$, we assign the observable

$$
\mathcal{O}(f)=\sum_{x_{+}} f\left(x_{+}\right) \mathcal{O}\left(x_{+}\right)
$$

Their transformation properties are

$$
\mathrm{U}(\mathrm{A}) \mathcal{O}(f) \mathrm{U}(\mathrm{A})^{\dagger}=\mathcal{O}\left(f \circ \mathrm{A}^{-1}\right)
$$

In this way we may establish contact between modular functions and forms of the finite Möbius group $\operatorname{SL}\left(2, \mathbb{F}_{p}\right)$, and conformal operators of definite conformal weight [24, 68, 69].

Once we have defined appropriate conformal operators, it is possible to calculate their correlation functions (and the identities these satisfy) in any state. The two- and threepoint functions can be determined from conformal invariance; the higher-point functions depend strongly on the quantum dynamics $[12,70]$.

In the next section we shall reconstruct bulk observables, when the boundary observables are known [71, 72].

\section{$5 \quad \operatorname{AdS}_{2}[N] / \mathrm{CFT}_{1}[N]$ coherent state holography}

In this section we present a new $\mathrm{AdS}_{2} / \mathrm{CFT}_{1}$ correspondence, $\operatorname{AdS}_{2}[p] / \mathrm{CFT}_{1}[p]$, based on the coherent states, of positive chirality, in the bulk and the boundary.A similar method can be applied to the subspace of negative chirality.

By $\mathrm{CFT}_{1}[p]$ we understand the quantum mechanics on the discrete, projective line, $\mathbb{R P}_{p}^{1}$, defined by the evolution operator $\mathrm{U}(\mathrm{A})$, for $\mathrm{A} \in \mathrm{SO}\left(2, \mathbb{F}_{p}\right)$. In analogy to the conformal quantum mechanics of ref. [12], the generator of this group corresponds to their "second" Hamiltonian, which has discrete spectrum. From the point of view of radial observers of 
the $\mathrm{AdS}_{2}$ near horizon geometry of an extremal black hole, this evolution corresponds to that of freely infalling observers [15-17].

To motivate the use of coherent states for the correspondence we notice the following:

The basic strength of the AdS/CFT correspondence relies on two important facts: first, the conformal boundary completion of the AdS space-time is very specific in selecting those boundary observables, which are appropriate for the reconstruction of those in the bulk-and this is holography. The second one is more constraining in that the AdS/CFT holography satisfies a new uncertainty principle, the IR/UV connection, which is a stringy effect. The higher the energy of the probe of a system on the boundary, in order to localize it, the bigger the distance form the boundary of the gravity dual system in the bulk. In the language of the stringy uncertainty principle, the higher the energy of the closed string state in the bulk, the larger the average length of the string: $\Delta x_{+} \Delta x_{-} \geq 1 / \alpha^{\prime}$.

In the light cone coordinates, $\left(x_{+}, x_{-}\right)$, on $\mathrm{AdS}_{2}, x_{+}$is parallel to the boundary and $x_{-}$is a measure of the distance from it. Strictly speaking, the appropriate quantity is $z \equiv 1+x_{+} x_{-}$, so, for fixed $x_{+}, x_{-} \rightarrow-1 / x_{+}$, when $z \rightarrow 0$.

In section 2 we observed that the variables $\left(x_{+}, x_{-}\right)$are appropriate holographic coordinates for the bulk, since they transform under the isometry group by Möbius, conformal, transformations:

$$
\begin{aligned}
& x_{+} \rightarrow \mathrm{A} x_{+} \equiv \frac{a x_{+}+b}{c x_{+}+d} \\
& x_{-} \rightarrow\left[\mathrm{A}^{-1}\right]^{\mathrm{T}} x_{-} \equiv \frac{d x_{-}-c}{-b x_{-}+a}
\end{aligned}
$$

Notice that $\left[\mathrm{A}^{-1}\right]^{\mathrm{T}}$ is the Fourier transform of $\mathrm{A} \in \mathrm{SL}\left(2, \mathbb{Z}_{N}\right)$,

$$
\left[\mathrm{A}^{-1}\right]^{\mathrm{T}}=\varepsilon \mathrm{A} \varepsilon^{\mathrm{T}}
$$

So $\left(x_{+}, x_{-}\right)$are conjugate variables, similar to position and momentum. Indeed, for $\mathrm{AdS}_{2}$, they represent time and length, promoting $\mathrm{AdS}_{2}$ into a stringy phase space. To saturate the stringy uncertainty principle we must employ the corresponding coherent states. In the bulk they have been defined as $\left|x_{+}, x_{-}\right\rangle$and the coordinates denote the center of the coherent state, while, on the boundary, as $\left|x_{+}\right\rangle$.

The bulk coherent states are the discrete analogs of the well-known wavelets, used in signal processing [73], which determine the spectrum of scales of a given signal as a function of position.

The boundary coherent states are, also, the discrete analogs of the usual coherent states of the harmonic oscillator (albeit on a different vacuum state) [36, 37].

We shall describe now the reconstruction method of the bulk observables (states) from appropriate boundary ones, using the wavelet representation and its properties.

Let us choose, for any value of the variable $x_{+}$, an independent variable, $x_{-}$, which takes values on the projective line, $\mathbb{R P}_{N}^{1}$, and define the state

$$
\left|\widetilde{x}_{-}\right\rangle \equiv F\left|x_{-}\right\rangle
$$


with $F$ the finite Fourier transform (3.19). Since $\left|x_{-}\right\rangle$is a boundary coherent state, we deduce that

$$
\widetilde{x}_{-}=-\frac{1}{x_{-}}
$$

In section 4 we constructed the chiral, scalar operators, $\mathcal{O}\left(x_{+}\right)$, It is obvious that the scalar operator,

$$
\widetilde{\mathcal{O}}\left(x_{-}\right) \equiv \mathcal{O}\left(\widetilde{x}_{-}\right)
$$

has conjugated transformation properties, i.e.

$$
\mathrm{U}(\mathrm{A}) \widetilde{\mathcal{O}}\left(x_{-}\right) \mathrm{U}(\mathrm{A})^{\dagger}=\widetilde{\mathcal{O}}\left(\left[\left(\mathrm{A}^{-1}\right]^{\mathrm{T}} x_{-}\right)\right.
$$

for any $\mathrm{A} \in \mathrm{SL}\left(2, \mathbb{F}_{p}\right)$.

We observe now that the composite operator,

$$
\mathcal{O}\left(x_{+}, x_{-}\right) \equiv \mathcal{O}\left(x_{+}\right) \widetilde{\mathcal{O}}\left(x_{-}\right)
$$

is a scalar operator in the bulk. Indeed,

$$
\mathrm{U}(\mathrm{A}) \mathcal{O}\left(x_{+}, x_{-}\right) \mathrm{U}(\mathrm{A})^{\dagger}=\mathcal{O}\left(\mathrm{A} x_{+},\left[\mathrm{A}^{-1}\right]^{\mathrm{T}} x_{-}\right)
$$

We shall use these operators to reconstruct Hermitian bulk scalar operators, so we must symmetrize the product in (5.7).

On the boundary, the operators $\mathcal{O}\left(x_{+}, x_{-}=-1 / x_{+}\right)=\mathcal{O}\left(x_{+}\right)$.

The reconstruction of the bulk operators from boundary data will be described next. The bulk/boundary correspondence in our construction is based on the fact that the Hilbert space of the bulk coincides with the Hilbert space of the boundary and carries the positive chirality component of the metaplectic representation of $\operatorname{SL}\left(2, \mathbb{F}_{p}\right)$. This places constraints on the algebra of observables on both sides of the correspondence.

Since both the bulk and boundary coherent states are overcomplete systems in the eigenspace of $P_{+}$, we get the relation

$$
\left|x_{+}, x_{-}\right\rangle=\frac{1}{d} \sum_{y_{+}} K\left(x_{+}, x_{-} \mid y_{+}\right)\left|y_{+}\right\rangle
$$

where the bulk/boundary propagator, $K\left(x_{+}, x_{-} \mid y_{+}\right)$, can be explicitly calculated:

$$
K\left(x_{+}, x_{-} \mid y_{+}\right)=\left\langle y_{+} \mid x_{+}, x_{-}\right\rangle
$$

From eqs. (4.6) and (4.31) we find that

$$
K\left(x_{+}, x_{-} \mid y_{+}\right)=((a-b \mu) \mid p)\left(-2 b^{\prime} \mid p\right)\left(\frac{1}{2}\left(y_{+}+\frac{1}{x_{-}}\right) \mid p\right)
$$

In this expression

$$
\begin{aligned}
& a=\frac{x_{+}}{\sqrt{x_{+}^{2}+1}} \\
& b=\frac{1}{\sqrt{x_{+}^{2}+1}} \\
& \mu=\frac{x_{+}-x_{-}}{1+x_{+} x_{-}}
\end{aligned}
$$


for the bulk coherent states and

$$
\begin{aligned}
a^{\prime} & =\frac{2 y_{+}}{1+y_{+}^{2}} \\
b^{\prime} & =\frac{1-y_{+}^{2}}{1+y_{+}^{2}}
\end{aligned}
$$

for the boundary ones.

The normalization constant, $d$, is defined through the overcompleteness relation of the boundary coherent states

$$
\sum_{y_{+}}\left|y_{+}\right\rangle\left\langle y_{+}\right|=d P_{+}
$$

Using eq. (4.31) we find that

$$
d\left\langle l\left|P_{+}\right| m\right\rangle=\sum_{y_{+}}\left\langle l \mid y_{+}\right\rangle\left\langle y_{+} \mid m\right\rangle \Rightarrow d=2
$$

The range of the bulk variables, $x_{ \pm}$, is determined by the light cone parametrization of the bulk, while the range of the boundary variable, $y_{+}$runs over the projective line, $\mathbb{R} \mathbb{P}_{N}^{1}$, i.e. $y_{+} \in\{0,1,2, \ldots, p-1\} \cup\{\infty\}$.

The correspondence between bulk/boundary observables can be constructed through the relation

$$
\left|x_{+}, x_{-}\right\rangle\left\langle x_{+}, x_{-}\right|=\frac{1}{d^{2}} \sum_{y_{+}, y_{-}} G\left(x_{+}, x_{-} \mid y_{+}, y_{-}\right) \mathcal{O}\left(y_{+}, y_{-}\right)
$$

The coefficient function, $G\left(x_{+}, x_{-} \mid y_{+}, y_{-}\right)$, can be determined from the bulk/boundary propagator

$$
G\left(x_{+}, x_{-} \mid y_{+}, y_{-}\right)=\frac{K\left(x_{+}, x_{-} \mid y_{+}\right) K^{*}\left(x_{+}, x_{-} \mid-1 / y_{-}\right)}{\left\langle y_{+} \mid-1 / y_{-}\right\rangle}
$$

The denominator is, in fact, a boundary/boundary propagator, whereas the numerator is the product of bulk/boundary, resp. boundary/bulk propagators.

\section{Summary and conclusions}

Before we summarize our results, let us review the conditions satisfied by our finite, discrete, model of the black hole near horizon geometry for the radial and temporal directions:

- In the space of all possible finite geometries we managed to single out the proposed type of modular discretization, by imposing the additional assumption of the existence of a holographic correspondence, to be satisfied through the replacement of $\mathrm{AdS}_{2}$ by $\operatorname{AdS}_{2}[N]$ and its boundary by $\mathrm{CFT}_{1}[N]=\mathbb{R P}_{N}^{1}$.

- Indeed, the finite geometry inherits the symmetry properties of its continuous counterpart (isometry group, coset structure and its quantum representations as well as bulk-boundary correspondence) albeit in a discretized disguise. 
- In the framework of the specific finite geometry it is very natural to choose as a model for the dynamics of probes the isometry group elements which, interestingly, possess strongly chaotic mixing properties. They are the well known Arnol'd cat maps, defined as Möbius transformations on the stereographic lightcone plane.

- Moreover, special properties of the modular representations guarantee the factorization with respect to the ultraviolet cut-off $N$. This is important for fast quantum information processing on the near horizon region. As we plan to show in forthcoming works the proposed framework is capable of providing an example for a mechanism for the saturation of the fast scrambling conjecture. [4, 55, 76, 77].

In the present work we have studied the modular discretization of the $\mathrm{AdS}_{2}$ geometry, $\operatorname{AdS}_{2}[N]$, and the ensuing classical and quantum dynamics of probes using generalized Arnold cat maps. We have demonstrated that our toy model is successful in realizing all of the properties which are considered key ingredients for a departure from semiclassical and local physics, namely those of non-locality, chaotic dynamics and fast quantum information processing.

With the discretization parameter, $N$, which provides both an ultraviolet and an infrared cutoff, the coset space nature of the $\mathrm{AdS}_{2}$ geometry "carries over" at the discretized level. The corresponding, effective Planck constant, $\hbar=2 \pi / N$ can be identified, also, with the non commutativity parameter of the quantum coset geometry [66, 67].

The strong arithmetic chaos of the Arnol'd cat map dynamics is inherited in a transparent way by the coset quantum states, which are the coherent states of $\operatorname{SL}\left(2, \mathbb{Z}_{N}\right)$. It is rather interesting that there is a correspondence between the bulk and the boundary states and observables of $\operatorname{AdS}_{2}[N]$; the latter belong to the discrete projective line, $\mathbb{R P}_{N}^{1}$. In a unique Hilbert space of finite dimension and given chirality, by using the overcompleteness of the corresponding coherent states of the bulk and the boundary, we provided a method to reconstruct the bulk states and observables from the corresponding boundary data. To this end we constructed the bulk-bulk, bulk-boundary and boundary-boundary propagators, which are invariant under the isometries of $\mathrm{AdS}_{2}[N]$. They are given by the overlap amplitudes between the corresponding coherent states.

These propagators realize the UV/IR connection between the bulk and the boundary scales, since the corresponding coherent states saturate the string uncertainty relation $\Delta x_{+} \Delta x_{-} \geq 1 / \alpha^{\prime}$

Our present work can be a basis for further extensions:

1. In the study of the $\operatorname{AdS}_{2}[N] / \mathrm{CFT}_{1}[N]$ correspondence for different representations of the discrete isometry group, $\operatorname{SL}\left(2, \mathbb{Z}_{N}\right)[68,69]$. In particular, it is interesting to study the modular discretization of the boundary conformal quantum mechanics of ref. [12]. It requires at the group level the definition of primary operators, their dimensions, as well as their fusion algebra.

2. Since the classical Arnol'd cat maps possess factorization in the parameter $N$ and strong chaotic properties by choosing $N=p^{n}$ where $p$ is a prime integer, we can construct the corresponding p-adic dynamics at both the classical and quantum levels. 
Indeed all of our amplitudes possess factorization properties. Therefore by taking their infinite product over $n$ from 1 to infinity it is possible to construct the corresponding p-adic amplitude [74, 75]. In recent works by Barbón et al. [76, 77]. it has been shown that ultrametric or p-adic structures of the Hilbert states black hole microstates which are supported by specific expander graphs guarantee the saturation of scrambling time bound for the black hole information processing [4].

3. Since the quantum Arnol'd cat maps possess factorization in the parameter, $N$, and strong chaotic properties [76, 77], they are also appropriate for the construction of quantum circuit models of deterministic chaos for the qubit information processing in black holes $[3,50,51,55,57,58]$. In analogy with the quantum circuit realization of Shor's factorization algorithm [78], it is expected that quantum circuits for the quantum Arnol'd cat maps will provide similar (exponential) improvements over their classical factorization properties and may saturate the scrambling bound [68, 69], as well.

\section{Acknowledgments}

MA and SN acknowledge the warm hospitality of the CERN Theory Division as well as of the LPTENS. We are thankful to A. Dabholkar, M.Porrati and E. Rabinovici for discussions and their constructive comments. EF is especially grateful to L. Alvarez-Gaumé, S.Ferrara and I. Antoniadis for making his stay at CERN such a rewarding experience. The research of EF is implemented under the "ARISTEIA-I" action (Code no. 1612, D.654) and title "Holographic Hydrodynamics" of the "operational programme education and lifelong learning" and is co-funded by the European Social Fund (ESF) and National Resources. The research of MA was supported in part by the General Secretariat for Research and Technology of Greece and the European Regional Develeopment Fund MIS-4483332-ORASY (NSRF 2007-13 ACTION, KRIPSIS).

Open Access. This article is distributed under the terms of the Creative Commons Attribution License (CC-BY 4.0), which permits any use, distribution and reproduction in any medium, provided the original author(s) and source are credited.

\section{References}

[1] G. 't Hooft, Black holes and quantum mechanics, Nucl. Phys. Proc. Suppl. 203-204 (2010) 155 [INSPIRE].

[2] J. Preskill, Do black holes destroy information?, in Proceedings of the International Symposium on Black holes, membranes, wormholes and superstrings, Woodlands, U.S.A., 16-18 January 1992 [hep-th/9209058] [INSPIRE].

[3] L. Susskind, L. Thorlacius and J. Uglum, The stretched horizon and black hole complementarity, Phys. Rev. D 48 (1993) 3743 [hep-th/9306069] [INSPIRE].

[4] Y. Sekino and L. Susskind, Fast Scramblers, JHEP 10 (2008) 065 [arXiv:0808.2096] [INSPIRE]. 
[5] A. Almheiri, D. Marolf, J. Polchinski, D. Stanford and J. Sully, An apologia for firewalls, JHEP 09 (2013) 018 [arXiv: 1304.6483] [INSPIRE].

[6] A. Sen, Extremal Black Hole Entropy, Lectures at the Asian Winter School, Kusatsu Japan (2012). Available at http://www.hri.res.in/ sen/asian12.pdf.

[7] B. Pioline, Corfu lectures on wall-crossing, multi-centered black holes and quiver invariants, PoS (Corfu2012) 085 [arXiv: 1304.7159] [INSPIRE].

[8] A. Sen, State Operator Correspondence and Entanglement in $A d S_{2} / C F T_{1}$, Entropy 13 (2011) 1305 [arXiv: 1101.4254] [INSPIRE].

[9] I. Mandal and A. Sen, Black Hole Microstate Counting and its Macroscopic Counterpart, Nucl. Phys. Proc. Suppl. 216 (2011) 147 [arXiv:1008.3801] [InSPIRE].

[10] A. Dabholkar and S. Nampuri, Quantum black holes, Lect. Notes Phys. 851 (2012) 165 [arXiv: 1208.4814] [INSPIRE].

[11] L. Susskind and J. Lindesay, An introduction to black holes, information and the string theory revolution: The holographic universe, World Scientific, Hackensack U.S.A. (2005).

[12] V. de Alfaro, S. Fubini and G. Furlan, Conformal Invariance in Quantum Mechanics, Nuovo Cim. A 34 (1976) 569 [inSPIRE].

[13] R. Kallosh, Black holes, branes and superconformal symmetry, in Corfu 1998, Quantum aspects of gauge theories, supersymmetry and unification, Corfu Greece (1998), pg. 138 [hep-th/9901095] [INSPIRE].

[14] R. Kallosh, Black holes and quantum mechanics, in Novelties in string theory, Goteborg Sweden (1998) [hep-th/9902007] [INSPIRE].

[15] R. Britto-Pacumio, J. Michelson, A. Strominger and A. Volovich, Lectures on superconformal quantum mechanics and multiblack hole moduli spaces, hep-th/9911066 [INSPIRE].

[16] P. Claus et al., Black holes and superconformal mechanics, Phys. Rev. Lett. 81 (1998) 4553 [hep-th/9804177] [INSPIRE].

[17] I. Bena, A. Puhm and B. Vercnocke, Non-extremal Black Hole Microstates: Fuzzballs of Fire or Fuzzballs of Fuzz?, JHEP 12 (2012) 014 [arXiv:1208.3468] [INSPIRE].

[18] K. Papadodimas and S. Raju, The Black Hole Interior in AdS/CFT and the Information Paradox, Phys. Rev. Lett. 112 (2014) 051301 [arXiv:1310.6334] [InSPIRE].

[19] K. Papadodimas and S. Raju, State-Dependent Bulk-Boundary Maps and Black Hole Complementarity, arXiv:1310.6335 [INSPIRE].

[20] K. Papadodimas and S. Raju, The unreasonable effectiveness of exponentially suppressed corrections in preserving information, Int. J. Mod. Phys. D 22 (2013) 1342030 [INSPIRE].

[21] J.L. Barbón and E. Rabinovici, Conformal Complementarity Maps, JHEP 12 (2013) 023 [arXiv: 1308.1921] [INSPIRE].

[22] M. Van Raamsdonk, A patchwork description of dual spacetimes in AdS/CFT, Class. Quant. Grav. 28 (2011) 065002 [INSPIRE].

[23] M. Van Raamsdonk, Evaporating Firewalls, arXiv:1307.1796 [INSPIRE].

[24] A. Terras, Finite Upper Half-Plane Graphs and their connection to Modular Forms, talk at Durham Symposium on Graph Theory and Interactions, Durham U.K., 15-25 July 2013. Available at http://www.math.ucsd.edu/ aterras. 
[25] L.M. Batten, Combinatorics of Finite Geometries, Cambridge University Press, Cambridge U.K. (1997).

[26] V.I. Arnol'd and A. Avez, Ergodic problems in classical mechanics, Benjamin, New York U.S.A. (1968).

[27] I.C. Percival and F. Vivaldi, Arithmetical properties of strongly chaotic motions, Physica D 25 (1987) 105.

[28] F. Vivaldi, Non-archimidean phenomena on the torus and lattice maps, http://www.maths.qmul.ac.uk/fv/research/talks.html.

[29] G. Athanasiu, E. Floratos and S. Nicolis, Fast quantum maps, J. Phys. A 31 (1998) L655 [math-ph/9805012] [INSPIRE].

[30] D. Ellinas and E. Floratos, Prime decomposition and entanglement measure of finite quantum systems, J. Phys. A 32 (1999) L63 [quant-ph/9806007] [InSPIRE].

[31] E. Floratos, The Heisenberg-Weyl Group on the $\mathbb{Z}_{n} \times \mathbb{Z}_{n}$ Discretized Torus Membrane, Phys. Lett. B 228 (1989) 335 [INSPIRE].

[32] J. Hoppe, Quantum theory of a massless relativistic surface and a two-dimensional bound state problem, Ph.D. Thesis, MIT, Cambridge U.S.A. (1982).

[33] T. Banks, W. Fischler, S. Shenker and L. Susskind, M theory as a matrix model: A Conjecture, Phys. Rev. D 55 (1997) 5112 [hep-th/9610043] [INSPIRE].

[34] J.H. Hannay and M.V. Berry, Quantization of linear maps on a torus - Fresnel diffraction by a periodic grating, Physica D 1 (1980) 267.

[35] J. Ford, G. Mantica and G.H. Ristow, The Arnol'd cat: Failure of the correspondence principle, Physica D 50 (1991) 493.

[36] R. Balian and C. Itzykson, Observations on finite quantum mechanics, C. R. Acad. Sc. Paris 303 I (1986) 773.

[37] G. Athanasiu and E. Floratos, Coherent states in finite quantum mechanics, Nucl. Phys. B 425 (1994) 343 [INSPIRE].

[38] G. Athanasiu, E. Floratos and S. Nicolis, Holomorphic quantization on the torus and finite quantum mechanics, J. Phys. A 29 (1996) 6737 [hep-th/9509098] [INSPIRE].

[39] O. Aharony, S.S. Gubser, J.M. Maldacena, H. Ooguri and Y. Oz, Large-N field theories, string theory and gravity, Phys. Rept. 323 (2000) 183 [hep-th/9905111] [INSPIRE].

[40] E. Witten, Anti-de Sitter space and holography, Adv. Theor. Math. Phys. 2 (1998) 253 [hep-th/9802150] [INSPIRE].

[41] L. Susskind and E. Witten, The Holographic bound in anti-de Sitter space, hep-th/9805114 [INSPIRE].

[42] J.M. Maldacena, Eternal black holes in anti-de Sitter, JHEP 04 (2003) 021 [hep-th/0106112] [INSPIRE].

[43] A. Strominger, AdS $S_{2}$ quantum gravity and string theory, JHEP 01 (1999) 007 [hep-th/9809027] [INSPIRE].

[44] J. Polchinski, L. Susskind and N. Toumbas, Negative energy, superluminosity and holography, Phys. Rev. D 60 (1999) 084006 [hep-th/9903228] [INSPIRE]. 
[45] A.J. Silberger, An elementary construction of the representations of $\mathrm{SL}(2, \mathrm{GF}(q))$, Osaka $J$. Math. 6 (1969) 329.

[46] G. Gibbons, Anti-de-Sitter spacetime and its uses, arXiv:1110.1206 [INSPIRE].

[47] I. Bengtsson, Anti-de Sitter space, http://www.fysik.su.se/ ingemar/Kurs.pdf.

[48] C. Patricot, A Group theoretical approach to causal structures and positive energy on space-times, hep-th/0403040 [INSPIRE].

[49] J-P. Gazeau, Coherent States in Quantum Physics, Wiley-VCH, Berlin Germany (2009).

[50] S.B. Giddings, Black holes, quantum information and unitary evolution, Phys. Rev. D 85 (2012) 124063 [arXiv:1201.1037] [INSPIRE].

[51] S.B. Giddings, Black holes, quantum information, and the foundations of physics, Phys. Today 66 (2013) 30 [INSPIRE].

[52] R. Bousso, B. Freivogel, S. Leichenauer, V. Rosenhaus and C. Zukowski, Null Geodesics, Local CFT Operators and AdS/CFT for Subregions, Phys. Rev. D 88 (2013) 064057 [arXiv: 1209.4641] [INSPIRE].

[53] T. Banks, Holographic Space-Time: The Takeaway, arXiv:1109.2435 [InSPIRE].

[54] D.N. Page, Time Dependence of Hawking Radiation Entropy, JCAP 09 (2013) 028 [arXiv:1301.4995] [INSPIRE].

[55] P. Hayden and J. Preskill, Black holes as mirrors: quantum information in random subsystems, JHEP 09 (2007) 120 [arXiv:0708.4025] [INSPIRE].

[56] L. Susskind, Black Hole Complementarity and the Harlow-Hayden Conjecture, arXiv: 1301.4505 [INSPIRE].

[57] S.G. Avery, Qubit models of black hole evaporation, JHEP 01 (2013) 176 [arXiv:1109.2911] [INSPIRE].

[58] D. Harlow and P. Hayden, Quantum computation vs. firewalls, JHEP 06 (2013) 085 [arXiv: 1301.4504] [INSPIRE].

[59] H. Weyl, The Theory of Groups and Quantum Mechanics, Dover, New York U.S.A. (1931).

[60] J. Schwinger, The geometry of quantum states, Proc. Nat. Acad. Sci. 46 (1960) 257.

[61] J. Schwinger, Unitary operator bases, Proc. Nat. Acad. Sci. 46 (1960) 574.

[62] J. Schwinger, Unitary transformations and the action principle, Proc. Nat. Acad. Sci. 46 (1960) 883.

[63] Y.I. Manin and M. Marcolli, Holography principle and arithmetic of algebraic curves, Adv. Theor. Math. Phys. 5 (2002) 617 [hep-th/0201036] [INSPIRE].

[64] A. Connes, Géométrie Non-commutative, Interéditions, Paris France (1990).

[65] A. Weil, Sur certains groupes d'opérateurs unitaires, Acta Mathematica 111 (1964) 143.

[66] H. Grosse and P. Prešnajder, The Construction on noncommutative manifolds using coherent states, Lett. Math. Phys. 28 (1993) 239 [INSPIRE].

[67] A. Jevicki and S. Ramgoolam, Noncommutative gravity from the AdS/CFT correspondence, JHEP 04 (1999) 032 [hep-th/9902059] [INSPIRE].

[68] M. Axenides, E.G. Floratos and S. Nicolis, Strong Arithmetic Chaos and the fast scrambling conjecture, in preparation. 
[69] M. Axenides, E.G. Floratos and S. Nicolis, Finite Conformal Quantum Mechanics, in preparation.

[70] C. Chamon, R. Jackiw, S.-Y. Pi and L. Santos, Conformal quantum mechanics as the CFT dual to $A d S_{2}$, Phys. Lett. B 701 (2011) 503 [arXiv:1106.0726] [INSPIRE].

[71] A. Hamilton, D.N. Kabat, G. Lifschytz and D.A. Lowe, Holographic representation of local bulk operators, Phys. Rev. D 74 (2006) 066009 [hep-th/0606141] [InSPIRE].

[72] K. Papadodimas and S. Raju, An infalling observer in AdS/CFT, JHEP 10 (2013) 212 [arXiv: 1211.6767] [INSPIRE].

[73] S. Mallat, A Wavelet Tour of Signal Processing, Academic Press, New York U.S.A. (2008).

[74] V. Vladimirov and I. Volovich, P-adic quantum mechanics, Commun. Math. Phys. 123 (1989) 659 [INSPIRE].

[75] P.G. Freund and M. Olson, p-adic dynamical systems, Nucl. Phys. B 297 (1988) 86 [INSPIRE].

[76] J.L. Barbón and J.M. Magán, Fast Scramblers And Ultrametric Black Hole Horizons, JHEP 11 (2013) 163 [arXiv:1306.3873] [INSPIRE].

[77] J.L. Barbon and J.M. Magan, Fast Scramblers, Horizons and Expander Graphs, JHEP 08 (2012) 016 [arXiv:1204.6435] [INSPIRE].

[78] P.W. Shor, Polynomial time algorithms for prime factorization and discrete logarithms on a quantum computer, SIAM J. Sci. Statist. Comput. 26 (1997) 1484 [quant-ph/9508027] [INSPIRE]. 